\title{
A Convergence Guaranteed Algorithm in Design of BJT Widlar Current Source Amplifiers
}

\author{
Jiecai Luo \\ Electrical Engineering Department \\ Southern University and A \& M College, P.O. Box 9969, \\ Baton Rouge, LA 70813 \\ jcluo@engr.subr.edu \\ Pradeep K. Bhattacharya \\ Electrical Engineering Department \\ Southern University and A \& M College, P.O. Box 9969, \\ Baton Rouge, LA 70813 \\ bhattach@engr.subr.edu
}

\begin{abstract}
In the course of microelectronics, Widlar current source amplifiers are very important part of the class courseware. Specially, in design of BJT Widlar current source amplifiers for the given current and extra resistor, a student must solve a nonlinear equation to get desired current output and this turns out to be a difficult step in design. In this short paper, a new convergence guaranteed algorithm to solve the nonlinear equation is developed, some simulation results are also provided. With this new algorithm, students can easily do their design.
\end{abstract}

\section{Introduction}

Microelectronics is intended as a core course in electronics for undergraduate electrical and computer engineering students. A majority of electronic circuits today are designed as integrated circuits, in which the entire circuit is fabricated on a single piece of semiconductor material. The integrated circuit may contain over a million semiconductor devices and other elements, and may perform many complex functions. The microprocessor is an example of such a circuit.

Initially, discrete transistor circuits were fundamental in learning basic knowledge for integrated circuits, and Widlar current source amplifiers are very important part of the class courseware. Specially, in design of BJT Widlar current source amplifiers for the given current and extra resistor, a student must solve a nonlinear equation to get desired current output and this turns out to be a difficult step in design. In this short paper, a new convergence guaranteed algorithm to solve the nonlinear equation is developed, some simulation results are also provided. With this new algorithm, students can easily do their design.

Proceedings of the 2003 ASEE Gulf-Southwest Annual Conference

The University of Texas at Arlington

Copyright (C) 2003, American Society for Engineering Education 
The rest of this paper is organized as follows: Section 2 describes the Widlar current source amplifier's design problem. Section 3 provides a new convergence guaranteed algorithm to design the Widlar current source amplifiers. Simulation results will be provided in section 4 . Some discussions will be given in the section 5 .

\section{The design problem statement for the Widlar current source amplifiers}

The current mirror [1-4] suffers from one deficiency: The mirrored current must always equal the reference current if matched devices are used. A more refined version of the current mirror, called the Widlar source, sets the mirrored current to a value smaller than $I_{\text {ref }}$ by using an extra resistor $R_{2}$ between the emitter $Q_{2}$ and the negative bus, as shown in figure 1 .

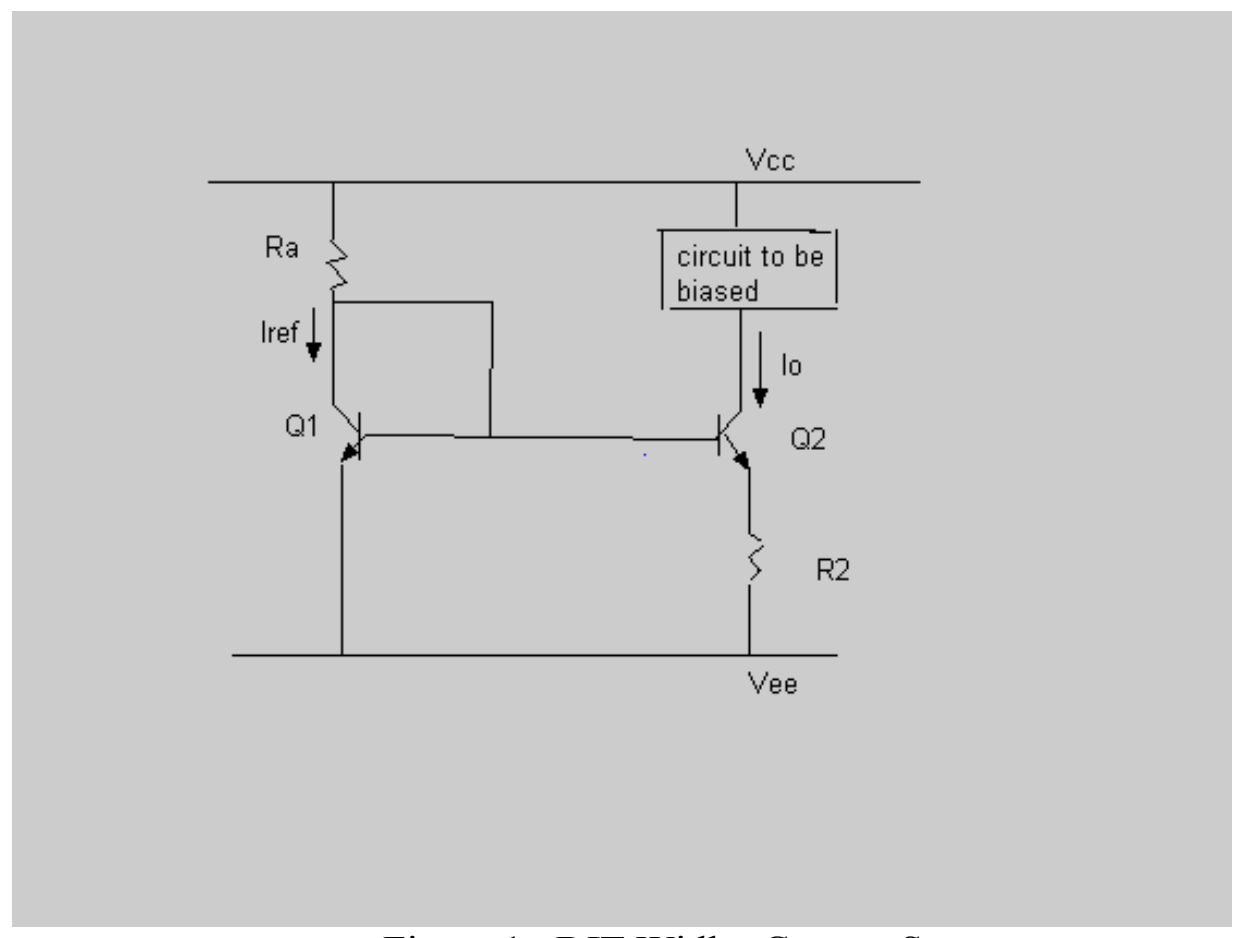

Figure 1: BJT Widlar Current Source

If $Q_{1}$ and $Q_{2}$ are perfectly matched, then the relationship among $I_{r e f}, R_{2}$ and $I_{o}$ (Widlar current) is

$$
I_{o}=\frac{\eta V_{T}}{R_{2}} \ln \frac{I_{r e f}}{I_{o}}
$$

Where $\eta$ is the emission coefficient, $V_{T}$ is the thermal voltage, and $V_{T}=25 \mathrm{mV}$ at room temperature. 
One of the design problems corresponding to the figure 1 is when $I_{r e f}, R_{2}$ are given, how to figure out $I_{o}$, before building the real BJT Widlar current source amplifier. To figure out $I_{o}$ is to solve the nonlinear equation (1) and this turns out to be a tough step for students. In the next section, a new convergence guaranteed algorithm to solve the nonlinear equation (1) is developed.

\section{A New Convergence Guaranteed Algorithm}

Traditionally, to solve the equation (1), necessary seven steps [1] are adopted as follow:

1. Input $I_{r e f}, R_{2}$;

2. Set $I_{o}$ to initial guess;

3. Choose iteration error margin $\Delta$;

4. New $I_{o}^{\prime}=\frac{\eta V_{T}}{R_{2}} \ln \frac{I_{r e f}}{I_{o}}$;

5. Is $\left|I_{o}-I_{o}^{\prime}\right|<\Delta$ ?

6. If the condition above is satisfied, then go to step 7, otherwise, go to step 4 again;

7. $I_{o}=$ Final answer.

When the algorithm given above is adopted, there are at least two problems: (1) it is not guaranteed that $I_{o}$ will converge to the real $I_{o} ;(2)$ Once it converges, it works very slowly.

About the second problem, we will see the results through the simulation in section 4 .

To overcome these two problems mentioned above, here is a new convergence algorithm developed, based on the two observations: (1) When $R_{2}=0, I_{r e f}=I_{o}$ and $I_{r e f} \geq I_{o} ;$ (2)

$\frac{d R_{2}}{d I_{o}}=-\frac{\eta V_{T}}{I_{o}^{2}} \ln \frac{I_{r e f}}{I_{o}}-\frac{\eta V_{T}}{I_{o}^{2}}<0$, which means when $R_{2}$ increases, $I_{o}$ decreases.

1. Input $I_{r e f}, N=0, R_{2}$ and $I_{o}=I_{\text {ref }}$;

2. Choose iteration error margin $\Delta$;

3. New $R_{2}^{\prime}=\frac{\eta V_{T}}{I_{o}} \ln \frac{I_{r e f}}{I_{o}}, I_{o}=I_{0}+\operatorname{sign}\left(R_{2}^{\prime}-R_{2}\right) *\left(\frac{I_{r e f}}{2^{N}}\right), N=N+1$;

4. Is $\left|R_{2}-R_{2}^{\prime}\right|<\Delta$ ?

5. If the condition above is satisfied, then go to step 6, otherwise, go to step 3 again;

6. $I_{o}=$ Final answer.

The Matlab code based on the 6 steps above is developed as follow:

\% This Matlab code is used to solve nonlinear equation

\% Io=eta*Vt/R2*ln (Iref/Io)

Proceedings of the 2003 ASEE Gulf-Southwest Annual Conference

The University of Texas at Arlington

Copyright (C) 2003, American Society for Engineering Education 


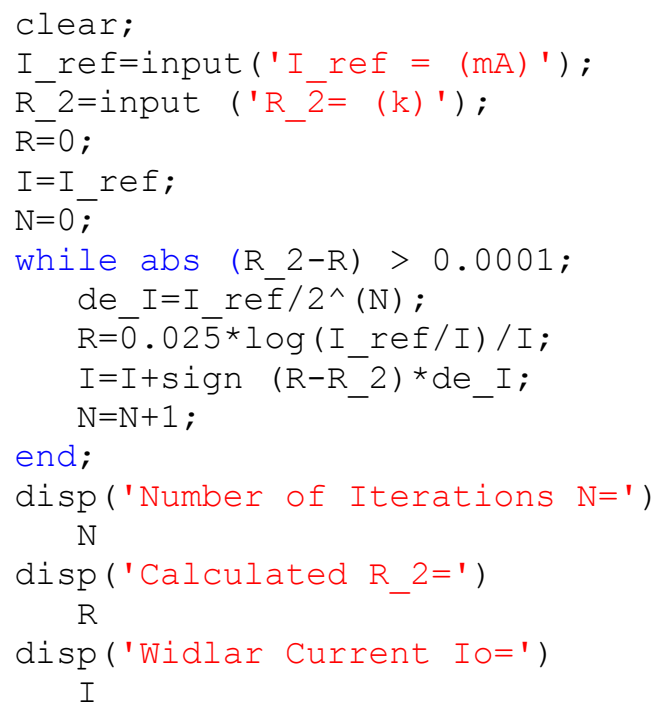

The simulation results for using new convergence algorithm are given in the next section. For the comparison, the simulation results by using traditional algorithm above for the same design problem are also provided.

\section{Simulation Results}

Let us consider the BJT Widlar current source amplifier design problem as follows:

In the circuit of Fig. 1, suppose that the values of $I_{r e f}=1.0 \mathrm{~mA}$. Find the value of $I_{o}$ if $R_{2}=1 \mathrm{k} \Omega$ (use the value $V_{T}=0.025 \mathrm{~V}$ and $\eta=1$ at room temperature).

The simulation results are shown in table 1 by using the new algorithm proposed above.

Table 1: Value of current through Widlar source obtained by new algorithm with $\Delta=0.0001$.

\begin{tabular}{|c|c|c|}
\hline Number of Iterations & $I_{o}(m A)$ & $R_{2}^{\prime}=\frac{\eta V_{T}}{I_{o}} \ln \frac{I_{r e f}}{I_{o}}(k \Omega)$ \\
\hline 0 & Initial guess 1.00 & 0 \\
\hline 19 & 0.0674 & 1.000 \\
\hline
\end{tabular}

For the comparison, the simulation results by using traditional iteration algorithm [1] for the same design problem are also given in table 2 .

Proceedings of the 2003 ASEE Gulf-Southwest Annual Conference

The University of Texas at Arlington

Copyright (C) 2003, American Society for Engineering Education 
Table 2: Value of current through Widlar source obtained by iteration with $\Delta=0.001$.

\begin{tabular}{|c|c|c|}
\hline Number of Iterations & $I_{o}(m A)$ & $I_{o}^{\prime}=\frac{\eta V_{T}}{R_{2}} \ln \frac{I_{r e f}}{I_{o}}(m A)$ \\
\hline 0 & Initial guess 1.00 & 0.00 \\
\hline 933 & 0.068 & 0.067 \\
\hline
\end{tabular}

By the comparison, the number of iterations of the new algorithm is much reduced.

\section{Summary and Conclusions}

In summary, a new convergence algorithm was developed here, and it will help the students to do their design. The new algorithm was based on the monotonic relationship between the extra resistor and the Widlar current, so that its convergence is guaranteed, and its efficiency has been shown in the section 4 . For any engineering design problem, if design involves solving some nonlinear equations, then the principle above can be used to solve the design problem.

\section{References}

1. Mark N. Horenstein, Microelectronic Circuits and Devices. Second edition, Prentice Hall, 1996.

2. Donald A. Neamen, Electronic Circuit Analysis and Design, second edition, McGraw-Hill Higher Education 2001.

3. M. Roden, G. Carpenter and W. Wieserman, Electronic Design from Concept to Reality. Fourth edition, Discovery Press, 2002.

4. Rashid, Microelectronic Circuits analysis and design, PWS Publishing Company, 1999.

JIECAI LUO

Jiecai Luo earned B. S. and M. S. in Electrical Engineering from Tongji University at Shanghai and HUST at Wuhan in China respectively, and PhD from University of Minnesota. He currently serves as an Assistant Professor of Electrical Engineering at Southern University, Baton Rouge. His research interests include control systems, optimal control and material science. He is a member of IEEE.

PRADEEP K. BHATTACHARYA

Pradeep Bhattacharya earned $\mathrm{PhD}$ in electronics in the faculty of Engineering from Indore University, Indore, India. He has worked at Microelectronics Center of North Carolina and did his research in solid state electronics in N. C. State University and MCNC from 1986-1990. He later worked as an Associate Professor of Electrical and Computer Engineering at LSU and now serves as a Professor in Southern University, Baton Rouge. He is currently the interim chair of Electrical Engineering Department at Southern University. His research interests are MEMS devices, sensors and nano technology.

Proceedings of the 2003 ASEE Gulf-Southwest Annual Conference

The University of Texas at Arlington

Copyright (C) 2003, American Society for Engineering Education 